\title{
Ph Salival como factor asociado a la caries dental
}

Salivary Ph as a factor associated

of Dental Caries

Fecha de Recepción

10 de marzo de 2017
O Ph Salivar como fator associado

de Cárie Dentária
Carolina Elizabet Barrios

Docente Auxiliar de la Cátedra Práctica

Clínica Preventiva I.

Email: cebarrios@odn.unne.edu.ar

Vilma Graciela Vila

Ex Profesora Titular de la Cátedra Práctica

Clínica Preventiva I.

Email:vgvila@odn.unne.edu.ar

Sandra Elena Martinez

Profesora Adjunta de la Cátedra Práctica

Clínica Preventiva I.

Email: semartinez@odn.unne.edu.ar

Alejandro J. Encina Tutuy

Docente auxiliar.

E-mail: jacoet@hotmail.com

\section{Resumen}

En la cavidad bucal existen múltiples factores que favorecen el desarrollo de caries dental. El presente estudio descriptivo, transversal tiene el propósito de conocer la relación entre las variaciones $\mathrm{pH}$ salival y presencia de caries en pacientes que asisten a la cátedra Práctica Clínica Preventiva II de la Facultad de Odontología.

La población de estudio estuvo constituida por 30 adolescentes de entre 13 y 26 años de ambos sexos que concurrieron durante el periodo marzo a diciembre de $201 \mathrm{I}$ para su atención. Se incluyeron aquellos clínicamente sanos, con estado nutricional normal y que no hayan consumido ningún tipo de alimento dos horas antes de la toma de muestra. Como criterios de exclusión se considero que posean enfermedades sistémicas, alteración en glándulas salivales y que puedan estar tomando alguna medicación anticolinérgica. A cada uno de ellos se le explico objetivos y métodos a utilizar verbalmente y escrito a través de un consentimiento informado, posteriormente se realizo una historia clínica completa , odontograma para establecer de esta manera el índice CPOD, consistente en observar las piezas dentarias careadas, perdidas y obturadas tomando como unidad el diente, siguiendo los criterios establecidos por la OMS. Subsiguientemente se coloco en piso de boca en contacto directo con la saliva la tira de prueba reactiva del $\mathrm{pH}$ se espero 5 minutos y se observo el cambio de color con el tiempo transcurrido. Finalmente se comparo el color de la 
almohadilla de prueba con el cuadro de colores normal. Este indicador colorido refleja el $\mathrm{pH}$ de la tira.

De acuerdo a las variables estudiadas, los resultados obtenidos en el índice de caries, son elevados no compatibles con salud, y en relación al $\mathrm{pH}$ la mayoría presenta un $\mathrm{pH}$ entre 6 y 7 manifestándose un alto índice de caries en personas que presentan un $\mathrm{pH} 5$.

\section{Palabras Clave}

Adolescentes, $\mathrm{pH}$, saliva, caries.

\section{Summary}

In the mouth cavity there exist multiple factors that favor the development of dental decay. The present descriptive, transverse study has the intention of determining the prevalencia of caries in relation to the salivary $\mathrm{pH}$ in patients who are present at the Practical chair Preventive Clinic the I Ind of the Faculty of Odontolgy. The population of study was constituted by 30 teenagers of between 13 and 26 years of both sexes that met during the period in March to December, $201 \mathrm{I}$ for his attention. Clinical healthy those were included, with nutritional normal condition and that have not consumed any type I feed two hours before the capture of sample. As criteria of exclusion I consider that they should possess systemic diseases, alteration in salivary glands and that could be taking some medication anticolinérgica. To each of them I him explain aims and methods to using verbally and writing across an informed assent, later I realize a clinical complete history, odontograma to establish hereby the index CPOD, consistent in observing the pieces dentarias compared, lost and plugged taking the tooth as a unit, following the criteria established by the who. Subsequently I place in floor of mouth in direct contact with the saliva the strip of test it reactivates of the $\mathrm{pH} I$ wait 5 minutes and I observe the change of color with the passed time. Finally I compare the color of the ink pad of test with the normal picture of colors. This colouring indicator reflects the $\mathrm{pH}$ of the strip.

For previously exposed it turns out to be to us significant to study the causal relations and the participation of multiple factors that act together and influence the development of the caries dental, bearing in mind the possibility of using to the saliva as a preventive method and of diagnosis.

Key Words

Flow, pH, saliva, caries

\section{Resumo}

$\mathrm{Na}$ boca existem múltiplos fatores que favorecem o desenvolvimento da cárie dentária. Este estudo transversal tem como objetivo determinar a prevalência de cárie em relação ao $\mathrm{pH}$ salivar em pacientes atendidos na cadeira de Prática Clínica Preventiva II da Faculdade de Odontologia.

A população do estudo consistiu de 30 adolescentes entre 13 e 26 anos de ambos os sexos que compareceram durante o período de Março a Dezembro de 201 I por sua atenção. Foram incluídos os clinicamente saudáveis, com estado nutricional normal e que não tenha consumido qualquer alimento duas horas antes da amostragem. Os critérios de exclusão foi considerado com doenças sistêmicas, alterados glândulas salivares e que pode tomar qualquer medicamento anticolinérgico. Para cada um deles eu explicar os objetivos e métodos a serem usados por via oral e por meio de um termo de consentimento informado, então fizemos uma história médica completa, o CPOD odontogram estabelecendo assim constituído observar dentes cariados, perdidos e encheu em um dente por, seguindo os critérios estabelecidos pela OMS. Posteriormente piso foi colocado em contato direto com a boca tira de teste de saliva é $\mathrm{pH}$ esperança reativa 5 minutos e mudança de cor observada com o tempo. Por fim, a comparação da cor da almofada de teste com a caixa de cor normal. Este indicador reflete a faixa de cor $\mathrm{pH}$.

Pelas razões expostas acima que acham importante estudar as relações causais e do envolvimento de múltiplos fatores agindo em conjunto e influenciar o desenvolvimento da cárie dentária, tendo em mente a possibilidade de usar a saliva como um método de diagnóstico e prevenção.

\section{Palavras Chave}

Fluxo, pH, saliva, cáries. 
REVISTA FACULTAD DE ODONTOLOGÍA ISSN No 1668-7280 - Vol. X No 1 - 2017
INVESTIGACIÓN

Ph salival como factor asociado a la caries dental

\section{Introducción}

La caries dental ha sido objeto de estudio desde el principio de la humanidad, ya que es una de las enfermedades más comunes en el hombre por lo tanto despertó la curiosidad de estudiosos que se dedicaban a buscar las causas que le daban origen. Esta enfermedad se produce por un desequilibrio del balance fisiológico de todos los factores y que van a determinar la composición del fluido de la placa en la superficie dental'.

La aparición y posterior progreso de la caries se debe a la intervención de tres factores primarios como son: la microbiota local representada por las bacterias acidógenas, el huésped representado por la saliva y los dientes, la ingesta de carbohidratos y el tiempo ${ }^{2,3}$.

Análogamente, existen otros factores a los que hace referencia Fejerskov, que involucran aspectos de salud pública como ser el flujo y densidad de la saliva, clase social, edad, hábitos y actitud del paciente 4 .

Como factor influyente de la caries, la saliva es un fluido que se origina en las glándulas salivales mayores y menores, el cual se produce de manera constante, permitiendo una acción limpiadora sobre las superficies de los tejidos duros y blandos de la cavidad bucal. Se encuentran además en su composición propiedades antibacterianas que se originan de factores inmunes específicos y no específicos que incrementan su poder anticariogénico, la misma posee una capacidad amortiguadora y neutralizadora de los ácidos producidos por los organismos cariogénicos o ingeridos a través de la dieta, permitiéndole mantener un $\mathrm{pH}$ relativamente constante, es también una fuente constante de calcio y fosfato, necesarios para la remineralización del esmalte ${ }^{5}$.

Es fundamental conocer los componentes de la saliva y sus funciones en el mantenimiento de la salud oral, la importancia de la saliva en el desarrollo de la enfermedad de caries y en la formación de la placa bacteriana. Las variaciones en el flujo salival pueden verse afectadas por múltiples factores fisiológicos y patológicos, de forma reversible o irreversible, juega un papel fundamental en el mantenimiento de la integridad de las estructuras bucales, en la vida de relación, en la digestión y en el control de infecciones orales ${ }^{6}$.
La saliva como alternativa para el diagnóstico, de algunas enfermedades, como elemento para monitorizar la evolución de determinadas patologías o la dosificación de medicamentos o drogas proporciona una vía prometedora. La accesibilidad en su obtención y la correlación positiva entre múltiples parámetros en el suero y en la saliva son algunas de las ventajas que ofrece como instrumento diagnóstico ${ }^{7}$.

Cuando el $\mathrm{pH}$ de la saliva que debería estar entre 7 a 7,4 disminuye y se mantiene en el tiempo empezamos a ver síntomas como caries de cuello, recesión gingival, milolisis, desmineralización en el cuello, manchas blanquecinas en el esmalte. Hay muchos estudios que han demostrado que en bocas con muchas caries, enfermedad periodontal el $\mathrm{pH}$ de la saliva es ácido8.

En la saliva tenemos un mecanismo buffers que intenta mantener el $\mathrm{pH}$ entre el 7 y 7,4 pero como este mecanismo en determinadas circunstancias se encuentra alterado, principalmente por la ingesta desproporcionada de alimentos ó bebidas con $\mathrm{pH}$ ácido, higiene bucal deficiente, poco control de placa bacteriana, presencia de policaries, enf. periodontal etc ${ }^{9}$.

La capacidad tampón de la saliva es un factor importante, que influye en el $\mathrm{pH}$ salivar $\mathrm{y}$ en el proceso de remineralización dental, siendo la concentración de bicarbonato su principal componente; se relaciona con el flujo salivar, ya que cualquier circunstancia que disminuya el flujo salivar tiende a disminuir su capacidad tampón e incrementa el riesgo de caries, juega un significativo rol en la preservación y mantención de la salud oral ${ }^{10}$. Diversos autores han indicado que una alteración del flujo salival es un factor clave en el desarrollo de caries, enfermedad periodontal e infecciones oportunistas". Además, una inadecuada función glandular de la secreción salival provoca un deterioro en el proceso de alimentación, dificultando la masticación, el paso de los alimentos al tracto digestivo, e incluso produce modificaciones en el habla'2.

El pH salival, por su parte, crea condiciones ecológicas bucales que mantienen el equilibrio medioambiental previniendo la aparición de patologías como la caries dental. Sin embargo, no se han descripto alteraciones del $\mathrm{pH}$ asociadas a cargas articulares excesivas o durante los TTM. Existe una relación reportada entre el flujo sali- 
val y el $\mathrm{pH}$ de la saliva debido a las variaciones en las concentraciones de bicarbonato y fosfato asociadas con los cambios volumétricos. De esta forma, se puede especular que alteraciones del flujo salival repercutieran en el $\mathrm{pH}$, de existir una asociación funcional entre los receptores articulares y los que regulan el flujo de saliva a nivel glandular's.

En el año 2004 Layna y col realizaron una investigación con el objetivo de determinar cómo influye el $\mathrm{pH}$ salival (determinado mediante el método de Snyder) en la incidencia de caries en niños de 6 a 13 años. En este estudio se observo la presencia de un $\mathrm{pH}$ acido en un porcentaje del $25 \%$ en comparación con el $15 \%$ de $\mathrm{pH}$ alcalino en los alumnos de ambas escuelas, estos resultados demuestran que en el $\mathrm{pH}$ acido una mayor predisposición a la prevalencia de caries, además que el $\mathrm{pH}$ salival es un factor predisponente para determinar el índice de caries ${ }^{14}$.

En el año 2005, Olayo et col determinaron el flujo, $\mathrm{pH}$ y la actividad peroxidasa de la saliva estimulada en 82 niños cubanos de ambos sexos con edades comprendidas entre 7 y II anos agrupados, de acuerdo al CPOD, en tres grupos (I: niños sin dientes afectados, II niños que presentaron $1 / 4$ dientes afectados, III niños que presentaron 7 o más dientes afectados) Donde hallo diferencia significativa para el $\mathrm{pH}$ salival entre los grupos I y III (grupo I: 8.0, grupo II: 7.7, grupo III: 7,5), también observo que el valor disminuye a mas piezas afectadas. Concluyeron que la diferencia obtenida para el $\mathrm{pH}$ salival entre los niños sin afectación y aquellos que presentaron 7 o más refuerzan los criterios que medio más acido es favorable para el desarrollo de caries ${ }^{15}$.

El objetivo del presente trabajo consistió en determinar la relación del $\mathrm{pH}$ salival y presencia de caries en pacientes que asisten a la cátedra Práctica Clínica Preventiva II de la Facultad de Odontología.

\section{Materiales y Método}

Población y Muestra: La población de estudio incluyo a pacientes de ambos sexos que asistieron a la Facultad de Odontología para su atención durante el periodo lectivo 20 I I. La muestra incluyo 30 pacientes en relación a la cantidad de pacientes que se atienden en la cátedra anterior- mente mencionada.

Criterios de Inclusión:

- Pacientes atendidos en la Facultad de Odontología

- Pacientes clínicamente sanos, con estado nutricional normal.

- Pacientes que no hayan consumido ningún tipo alimento dos horas antes de la toma de muestra.

Criterios de Exclusión

- Pacientes con enfermedades sistémicas como hipertiroidismo, diabetes, anemia, nefritis, etc.

- Pacientes con alteración en glándulas salivales.

- Pacientes que puedan estar tomando alguna medicación anticolinérgica, antidepresivos tricíclicos, antconvulsionantes, antihistamínicos, antihipertensivos, antieméticos, anti parkinsonianos, antiespasmódicos, anorexigenos, diuréticos, descongestionantes, expectorantes, relajantes musculares y psicotrópicos.

El tipo de estudio descriptivo transversal.

\section{Procedimiento para la selección de pacientes}

Participaron alrededor de 30 pacientes a quienes se les realizo una historia clínica completa en aquellos con aparente buen estado de salud general.

A estos pacientes se les explico objetivos y métodos a utilizar verbal y escrito a través de un consentimiento informado, posteriormente a los pacientes que cumplían con ciertos requisitos como presentar alguna enfermedad sistémi$\mathrm{ca}$, ni consumir algún medicamento que pueda influir en el resultado de las variables y que no hayan consumido ningún alimento algunas dos horas antes de la toma de muestra se confecciono la historia clínica completa.

\section{Procedimiento para el índice CPOD}

Se realizo en cada uno de los pacientes el odontograma para establecer el índice CPOD, consistente en observar las piezas dentarias careadas, perdidas y obturadas tomando como unidad el diente, siguiendo los criterios establecidos por la OMS. 
Procedimiento para determinar el $\mathrm{pH}$ salival La saliva tiene una capacidad de neutralizar ácidos o mejor dicho de amortiguar las variaciones de $\mathrm{pH}$. Esta capacidad esta basada en varios sistemas como el sistema de fosfato y el sistema de bicarbonato - ácido carbónico. En la saliva no estimulada, la concentración de fosfato inorgánico es bastante mas alta que la concentración del sistema bicarbonato - ácido carbónico. El sistema bicarbonato - ácido carbónico es el más importante en la saliva estimulada debido a su mayor concentración. Se utilizara el método simplificado se ha desarrollado bajo el nombre de Dentobuff® Strip System, se procede a colocar una tira reactiva en el piso de boca en contacto con la saliva, se espero cinco minutos y se observo el cambio de color con el tiempo transcurrido. Finalmente se compara la tira de color con el cuadro de colores normal, este indicador colorido refleja el $\mathrm{pH}$ de la tira.

\section{Resultados y Discusión}

La muestra estuvo conformada por 30 adolescentes de ambos sexos, con una edad promedio de 20.86 . El $22 \%$ de la población tiene alrededor de 15 años, el $56 \%$ tiene entre 16 y 20 y $22 \%$ restante tiene entre 21 y 22 años. Gráfico I La condición bucal de la muestra obtenida (estado gingivo - dentario) de los pacientes seleccionados, se reflejo con los valores arrojados por los índices de caries, gingival y de placa. El $46 \%$ de la muestra presento valores muy altos de índice de caries (CPO) según parámetros establecidos por la OMS (más 4.5 a 6.5). Se obtuvieron valores de $\mathrm{pH}$ que variaron entre 5 y 7 , siendo el intervalo de referencia normal 6,5. El 34\% presento $\mathrm{pH}$ de 7 mientras que el $64 \%$ mostraron entre 5 y 6. Gráfico 2

Velázquez, Diego y col. en un estudio establecieron que la relación del $\mathrm{pH}$ salival con los hábitos bucales, dieta y placa bacteriana que pueden influir en la presencia de caries dental en los niños de la escuela San José, Norte. No se halló ninguna relación entre la presencia de hábitos bucales y el valor del $\mathrm{PH}$ salival.

Estudios realizados por Aguilera y col. en un grupo de I50 niños de ambos sexos de una escuela primaria de la zona urbana de la ciudad de Zacatecas encontraron una prevalencia de caries dental de $56 \%$, así mismo se detectó una correspondencia entre los niveles de S. mutans $y$ el CPOD.

\section{Conclusiones}

De acuerdo a las variables estudiadas, los resultados obtenidos en el índice de caries, son elevados no compatibles con salud, y en relación al $\mathrm{pH}$ la mayoría presenta un $\mathrm{pH}$ entre 6 y 7 manifestándose un alto índice de caries en personas que presentan un $\mathrm{pH} 5$.

La caries dental es una enfermedad multifactorial, que precisa para su desarrollo de la interacción de factores como la resistencia del huésped, las relaciones microbianas, las características de la saliva y del sustrato, así como el tiempo para actuar. La evidencia proporcionada a nivel internacional sugiere que uno de los factores a considerar es la saliva cuyos componentes no solamente favorecen la prevención de caries, sino también pueden ser utilizados como herramienta de diagnóstico ${ }^{18}$. 
Gráfico I. Distribución de las distintas edades de los individuos incluidos en el estudio.
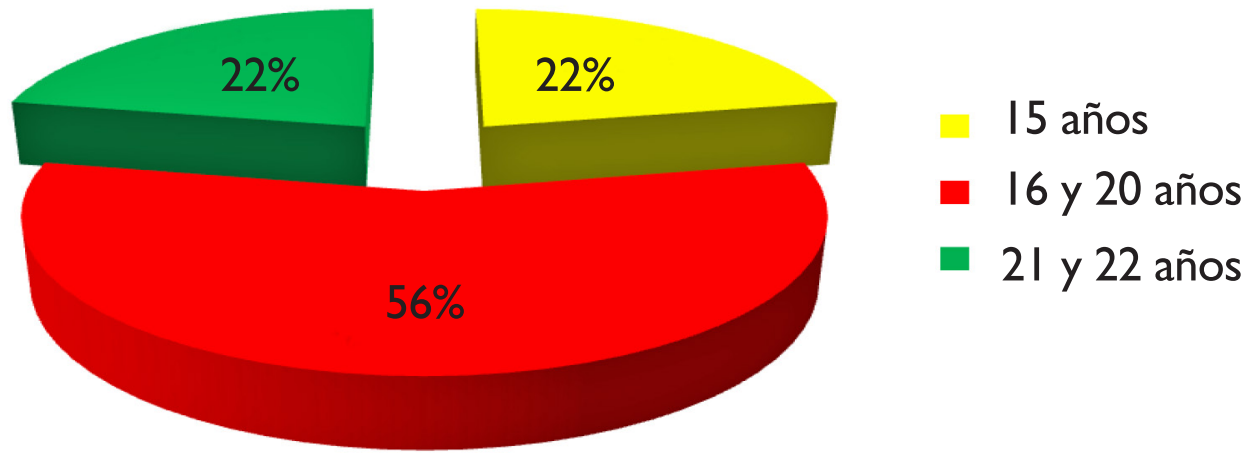

Gráfico 2. Relación entre pH y Caries.

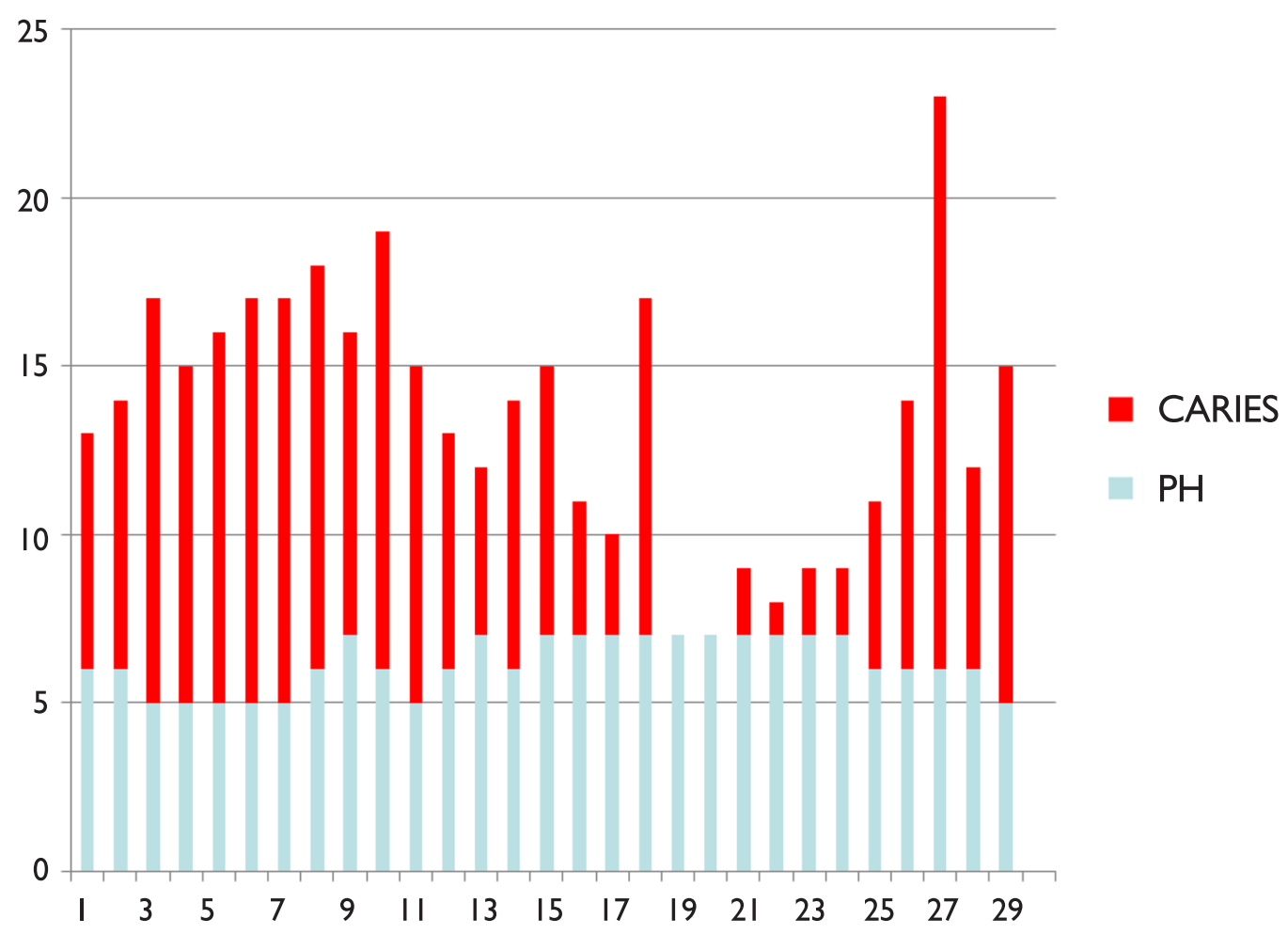


REVISTA FACULTAD DE ODONTOLOGÍA

ISSN No 1668-7280 - Vol. X No 1 - 2017
INVESTIGACION

Ph salival como factor asociado a la caries dental

\section{Bibliografía}

I. Romero H .M., Hernández Y. Modificaciones del pH y flujo salival con el uso de aparatología funcional tipo Bimler. Revista Latinoamericana de Ortodoncia y Odontopediatría [revista en Internet]. Marzo 2009. [acceso 19 de marzo 2010]; disponible en: http:// www.ortodoncia.ws/publicaciones/2009/art6.asp.

2. Loyo Molina, Kenny, Balda Zavarce, Rebeca, Gonzalez Blanco, Olga et al. Actividad Cariogenica y su Relación con el Flujo Salival y la Capacidad Amortiguadora de la Saliva. Acta odontol. venez. [Online]. dic. 1999, Vol.37, no.3 [citado 18 Agosto 20II], p.I0-17. DisponibleenWorldWideWeb:<http://www.scielo. org.ve/scielo.php?script=sci_arttext\&pid=S000 I 6365 I999000300003\&lng=es\&nrm=iso >. ISSN $0001-6365$

3. González, M.; Ledesma, C.; Banderas, J. A. Saliva y cavidad bucal. Parte I: Glándulas salivales: mecanismos fisiológicos de la secreción salival. Pract Odontol 2008; I5 (6): 7-I.

4. Denny, $\mathrm{P}$ and Cols. Age- related changes in mucins from human whole saliva. J Dent Res.2010;70 (10): I320-1327.

5. Guías Prácticas de Estomatología. Editorial Ciencias Médicas. La Habana, 2007. pp. 23 - 47.

6. Oliveri, P.; Mari Campo, E.; Adami, J.; Zubillaga M.; Boccio, J.; et. al. Identificación de la presencia de Helicobacter pylori (Hp) en la cavidad oral mediante un método de análisis radiométrico en saliva. Diagnóstico. 2008 Enero; IX (88). (Disponible en http://www. diagnostico.com.ar./diagnos-tico/dia088/dhpo88.htm).

7. Gonzalez, M.; Banderas, J. A.; Raya, C.; Baéz, A.; Belmont, R. Quantification of lead, cadmium and chomium by sialochemistry. Salud Pública Mex. 2007 May-Jun; 39(3):I79-18. I8. JANÉ SANTAMARÍA, M.; VAREA CALDERÓN, V.; MUÑOZ ALMAGRO, M. C. Estudio de la placa dental en la infección por Helicobacter pylori. Anales Españoles de Pediatría. 1999; 50 (3): 244-246.

8. Banderas, J. A; González, M. Saliva y cavidad bucal. Parte II: Proteínas salivales: funciones biológicas en el mantenimiento de la homeostasis bucal. Pract Odontol 2007; 15 (7): I3-20.
9. Krasse, B. O. Salivary examination. Caries risk. Chicago: Quintessence Publishing Co., Inc.2005:4l-44. Los nuevos métodos para la detección precoz del cáncer. La Tercera. Ciencia y Salud. Chile. (Disponible en http://www.tercera.cl/diario/2000/03/I7/t17.25.3a. CYS.NUE-VOS.html.)

10. Mandel ID. Relation of saliva and plaque to caries. J Dent Res Supplement to N². Vol 53, 2007.

II. Fejerskov O. Concepts Of. dental caries and their 4 . consequences for understanding the disease. Community Dent Oral Epidemiol 2005; 25: 5-12.

12. Dye BA, Tan S, Smith V, et al. Trends in oral health status; United States 1988-1994 and 1999-2004. National Center for Health Statistic. Vital Health Stat II 2007; (248): I-92.

13. Reich E. Trends in caries and periodontal health epidemiology in Europe. Int Dent J 2007; 5I: 392-8.

14. INTERNATIONAL CARIES DETECTION \& ASSESSMENTSystem Coordinating Committee.The International Caries Detection And Assessment System(ICDAS II). Workshop sponsored bythe NID$C R$, the ADA, and the InternationalAssociation For Dental Research. Available at: https://www.icdas.org. Accessed February, 2010. [Criteria Manual For The InternationalCaries Detection And AssessmentSystem (ICDAS II)].

15. Longbottom C, Huysmans MC, Pitts N, et al. Glossary of key terms. MonogrOral Sci 2009; 2I: 209-16.

16. Pitts NB, Stamm J. ICW-CCT statements. J Dent Res 2004;83(Speciallssue C): 125-8.

17. Featherstone JD, Domejean-Orliaguet $S$, Jenson L, et al. Caries risk assessment in practice for age 6 through adult. J Calif Dent Assoc 2007; 35(I0): 703-13.

18. Fontana M, Young D, Wolff M. Evidence based caries riskassessment and management. Dent Clin North Am 2009.

19. Fontana M, Zero D. Assessing patients' caries risk. J AmDent Assoc 2006; I 37 (9): I23 I - 40.

20. Jablonski-Momeni A, Stachniss V, Ricketts DN, et al. Reproducibilityand accuracy of the ICDAS-II for detection of occlusalcaries in vitro. Caries Res 2008; 42: 79 - 87.

21. Perejoan M. Sustitutos de la saliva. Revista Española de Estomatología, 2007; 326-332. 